\title{
Day-ahead Unit Commitment Considering of Wind Power and Bilateral Energy Contract
}

\author{
Liu Fang ${ }^{1}$, Luo Zhiqiang, Sun Zhen ${ }^{1}$, Liu Cong ${ }^{1}$, Liu Lihua ${ }^{2}$,Yang Junfeng ${ }^{2}$ \\ 1.China Electric Power Research Institute, Haidian District, Beijing 100192, China; \\ 2.State Grid Corporation of China, Xicheng District, Beijing 100031, China
}

\begin{abstract}
Keywords:unit commitment; wind power; bilateral transaction; contract reduction; electricity
\end{abstract} market.

\begin{abstract}
The operation of power system is transforming from traditional dispatching mode to market, and coupled with the uncertainty of intermittent energy, day-ahead electric power and energy balance will be a multi-objective coordination problem of new energy consumption, contract execution, grid security and generation economy in market transition phase. Studying day-ahead power balance coordination optimization model under new conditions is important to promote the consumption of wind power and ensure the fair execution of transactions. This paper used confidence interval method to model the uncertainty of wind power, established objective function and constraints to ensure the execution of contracts, and considered grid security and generation economy to establish the multi-objective coordinated unit commitment model. The paper used a test system to study the effect of confidence intervals on unit portfolio reliability and contract completion rate, to verify the validity of confidence intervals and fairness of allocation of reductions among power plants, to study the effect of power flow constraints on contract execution and the interplay among wind energy consumption, grid congestion, and contract execution. Results show that power plants reduce contracts according to their reduction factors, it will inhibit contract execution when wind power is small scale and causes a positive power flow in the blocking line, , resulting in a 61.15\% higher contract reduction than that without wind power; it will promote contract execution, when wind power causes reverse power flow in blocking line, leading to a 42.73\% down in contract reduction compared to no wind power. It shows that the unit commitment model is effective in wind power consumption, contracts fairly execution, power grid security and the economy of power generation.
\end{abstract}

\section{Introduction}

Power industry in China is facing new circumstances as the rapidly development of new energy and the guidance and propelling of power direct-purchasing. Power dispatching is facing challenges from different aspects such as new energy consumption, execution of bilateral transactions, accomplishment of Sangong standard and the security of power grid. Conventional energy should provide adjustable condition for new energy consumption for the stochastic volatility of wind and solar generation. Bilateral transactions should be executed as far as possible by means of fixing certain generation power. The new energy consumption needs the coordination of thermal generation, so that the consumption may have impact on the execution of bilateral transaction to a certain degree. The constraints of transmission lines and cross section would make the interaction between new energy consumption and bilateral transaction execution more complicated.

Two typical power markets throughout the world are PJM Electricity Market and British Electricity Market. In PJM, it is centralized bidding in day-ahead market and generation plants make step-wise offers. The clearing process consider plant offers only despite of the execution of bilateral transactions[1-3]. In Britain, power curve is submitted on both supply and user sides under bilateral transactions. The power curve needs to be executed and the balance of power system is implemented by a balancing mechanism[4].

There have been several achievements in study on bilateral transactions and wholesale competitive market[5-8] in China, among which achievements about quantitative analysis of 
bilateral transaction are very rare and most of them are focused on problems of pricing theory, game model, optimization transaction strategy and impact of bilateral transactions on power market,etc[9-13] , and the experience presentation about mode comparison of bilateral transaction market abroad[14-17]. Now as the deepen reformation is taken places in China power industry, there lacks of studies on the power balancing problem considering both new energy consumption and transaction execution.

Power industry in China is transiting to an open market. Neither Centralized Bidding nor Balancing Mechanism is applicable. In the present market, there are bilateral transaction contracts as well as planning power. The execution of the two different types of power is complicated. System operators need to take the bilateral transaction into consideration when scheduling day-ahead generation. Also, new energy consumption, power grid security and generation economy also need to be considered. Power balancing in new circumstances is facing challenges from more aspects than today. New energy consumption, transaction execution and power grid security are objectives need to be coordinated in power balancing. To solve the problem above, a day-ahead scheduling method is provided in this paper. Wind power consumption is priority ensured according to China's full protection of wind power purchase policy. Then the bilateral transaction should be executed as far as possible. When contracts must need to be cut, allocate the reduction quantity among generation plants fairly. On that basis, day-ahead plan can be scheduled considering power flow constraints.

\section{Multi-objective Coordination Strategy}

Day-ahead power balance coordination and optimization should take new energy consumption, bilateral transaction execution and grid security into consideration. On the basis of security constraints, wind power consumption has priority. Bilateral transaction and Sangong power should be executed as far as possible considering generation economy. To achieve objectives above, day-ahead scheduling should satisfy the following requirements: 1) Improve the reliability of unit commitment. When wind power fluctuates randomly in actual running, power system should have sufficient capacity to ensure the consumption of wind power. 2) Take security, transaction execution and generation economy into consideration. Find the unit commitment that costs least meanwhile transaction executes the most and wind power consumption is ensured.

In this paper, the uncertainty of wind power generation is estimated by confidence interval method[18]. Wind power generation output within a certain confidence interval can be consumed totally in regardless of generation cost. The confidence interval is confirmed based on wind power prediction.

Since there is no difference essentially between bilateral transaction power and Sangong power for generation plant, the two components of power implement is determined by the order of settlement. The two components of power are combined during optimization. The optimize objective is to maximum the total amount of the implement of power contracts, and the contract completions of each plant are the same. To minimum the contract reduction is also one of the objectives in optimization. Constraints related to contract power are also considered to maximum the execution of power in contract.

\section{Distribution of Contract Reduction}

To ensure fairness, when contracts need to be reduced, contracts of each plant are reduced in accordance of contract power proportion. If the bilateral contracts are energy contracts, the reduction factor is calculated in function (1). Contract energy and Sangong energy are combined in this function. Contract reduction can be distributed fairly among generation plants using this function.

$$
H X_{i}=\frac{H S_{i}}{\sum_{i} H S_{i}}
$$


In this function, $H X_{i}$ is the contract reduction factor for plant $i, H S_{i}$ is the total amount of bilateral and Sangong contract power for plant $i$.

The completion rate of contracts is of vital interest to generation plant. Generation companies have high requirement for the fairness of contract implement. Meanwhile, they are also subjected to accomplish the contract that costs least. The fairness of contract reduction distribution can be realized with reduction factor. However, the generation economy can be accomplished with the economy objective when distribute the contract reduction among generation units.

\section{Day-ahead Balancing Modelling-objective Functions}

If generation cost is the only objective when scheduling, the optimized result cannot guarantee the execution of contracts. In the same way, if contract execution is the only objective, the trading volume may allocate among the generation units arbitrarily. The execution result may not be economically optimal. If take multi-objectives of both contract execution and generation cost, the optimized result could take both contract execution and generation economy into consideration.

To minimum the reduction of contracts and minimum the generation cost, here introduce a penalty factor MF. First, guarantee the execution of contracts. Under this premise, units with better economy have output priority. So that the optimization result can reduce the generation cost and execute contracts as far as possible. The objective function is:

$$
\min \Delta S * M F+C(P)
$$

In which:

$$
\begin{gathered}
\Delta S=\sum_{j=1}^{H} S_{j, 0}-\sum_{i=1}^{N} \sum_{t=1}^{T} P_{i, S, t} \\
C(P)=\sum_{i=1}^{N} \sum_{t=1}^{T}\left(f_{i, t}^{0}+S_{U i, t}^{0}+S_{D i, t}^{0}\right)
\end{gathered}
$$

In this function, $\mathrm{H}$ is the total amount of generation plants. $\mathrm{N}$ is the total amount of thermal generation units. $\mathrm{T}$ is the time period. $\Delta S$ is the total reduction of contracts. $S_{j, 0}$ is the total energy of bilateral and Sangong contracts for plant $j$ (MWh). $P_{i, S, t}$ is contract output(MW). MF is the penalty factor and the bigger of $M F$, the higher of completion rate for contract execution. $C(P)$ is the cost of thermal generation(元). $f_{i, t}^{0} 、 S_{v i, t}^{0} 、 S_{D i, t}^{0}$ is respectively the operation cost, the start-up cost and shut-down cost of thermal generation unit $i$ at time $t$.

\section{Day-ahead Balancing Modelling-constraints}

Constraints here are the wind power prediction and the confidence interval limits of wind power. The former constraint is used to guarantee the security of power system and consumption of wind generation in the forecast scenario. The latter is used to improve the stability of unit commitment and to guarantee a sufficient coordinate capacity in power balancing when the wind power outputs fluctuate within the confidence interval.

\section{Constraints of wind power forecast.}

The constraints of wind power forecast are to guarantee the execution of transactions and generation economy in the forecast scenario. Hence the constraints include the constraints of power balancing, the running limits of thermal units, the related constraints of contacts, power flow constraints and reserve constraints etc. The constraint functions are:

$$
\begin{gathered}
\sum_{i=1}^{N} P_{i, t}+\sum_{j=1}^{W} P_{w j, t}=L_{t} \\
\Delta S_{j}=S_{j, 0}-\sum_{i \in N_{j}} \sum_{t=1}^{T} P_{i, S, t}, \quad j=1,2, \cdots, H
\end{gathered}
$$




$$
\begin{gathered}
\Delta S_{j}=S X_{j} \bullet \Delta S \\
P_{i, t}=P_{i, S, t}+P_{i, z, t}, \quad i=1,2, \cdots, N \\
P_{i, \min } I_{i, t} \leq P_{i, t} \leq P_{i, \max } I_{i, t} \\
\boldsymbol{k}(\boldsymbol{P}, \boldsymbol{I})=0 \\
\boldsymbol{y}(\boldsymbol{P}, \boldsymbol{I})=0 \\
\sum_{i=1}^{n}\left|A_{l i} P_{i, i n}\right| \leq F_{l, \max }, \quad l=1,2, \cdots b \\
\boldsymbol{I} \in(0,1), \Delta S_{j} \geq 0, P_{i, S, t} \geq 0, P_{i, z, t} \geq 0
\end{gathered}
$$

In this function, $H$ is the amount of generation plants. $N$ is the total number of thermal generation units. $W$ is the total amount of wind plants. $P$ is the output of thermal units in the wind forecat senerio, of which $P_{i, t}$ is the planning output for unit $i$ at time $t(\mathrm{MW})$. I is the running state of thermal units, of which $I_{i, t}$ is the running state for unit $i$ at time $t(\mathrm{MW}) . P_{w j, t}$ is the forecast power for wind power plant $j$ at time $t(\mathrm{MW}) . L_{t}$ is the system load at time $t(\mathrm{MW}) . \Delta S_{j}$ is the reduction amount of contracts for plant $j(\mathrm{MWh}) . S_{j, 0}$ is the summation of bilateral and Sangong energy for plant $j(\mathrm{MWh}) . \quad P_{i, S, t}$ is contract power of unit output and $P_{i, z, t}$ is the other composition output(MW). $S X_{j}$ is the factor of contract reduction for plant $j$ and $\Delta S$ is the total amout of contract reduction(MWh). $N_{j}$ is the serial number for plant $j . P_{i, \text { max }} 、 P_{i, \text { min }}$ are the upper and lower limit of thermal units(MW). $n$ is the number of nodes and $b$ is the number of branches. $A_{l i}$ is the sensitivity factor of net power injection of node $i$ to transmission line $l$. $F_{l, \text { max }}$ is the upper limit of power flow at line $l(\mathrm{MW}) . P_{i, i n}$ is the net power injection to nod $i(\mathrm{MW})$.

Function (3-a) is the power balancing constraint considering wind power forecast. Function (3-b) is the contract reduction for plant $j$. Function (3-c) is the relationship between the contract reduction for plant $j$ and the total contract reduction of the whole system. Function (3-d) is the decomposed constraint for power output of units. Function (3-e) is the output limitation constraint. Function (3-f) and the inequation (3-g) are the basic constraints, including the constraints related to the startup and shutdown constraint of thermal units, the minimum boottime and downtime constraints, the up-rotation and down-rotation reserve constraints and ramping constraints. Function (3-h) is the power flow constraint for transmission line.

The reduction among power plants should be coordinated. So the constraint of relationship between the contract reduction of power plants and the total reduction amount of power system is listed in the constraint functions. The non-negative constraint for contract reduction is used to prevent that the total reduction amount might be negative which counteracted the generation cost. What power plants really concentrate on is the implement of contracts. However, the contracts have to be reduced among the generation units due to the economist of units. The objective to minimum the generation cost may reduce generation cost and to maximum the implement of contracts at the same time.

\section{Constraints of confidence interval.}

Constraint on the wind forecast confidence interval takes the uncertainty of wind power into consideration. The constraints include power balancing limits based on the confidence interval of wind power, and running limits of thermal units, reserve limits, power flow limits and constraints on coupled coordinate capacity of the upper and lower confidence interval.

Constraints on confidence interval are used to improve the stability of unit commitment. That is to guarantee sufficient coordinate capacity in real time running of power system. Constraints on 
contract execution are not considered here for the reason that unit commitment is aimed to ensure the feasibility of unit composition in the worst condition.

- Basic and power flow constraints based on confidence interval

According to the distribution model of wind power prediction, the confidence interval of wind power can be get[19]. The constraints established according to confidence interval are:

$$
\begin{gathered}
\sum_{i=1}^{N} P_{i, t}^{s}+\sum_{j=1}^{W} P_{w j, t}^{s}=L_{t} \\
P_{i, \text { min }} I_{i, t} \leq P_{i, t}^{s} \leq P_{i, \text { max }} I_{i, t} \\
\boldsymbol{k}\left(\boldsymbol{P}^{s}, \boldsymbol{I}\right)=0 \\
\boldsymbol{y}\left(\boldsymbol{P}^{s}, \boldsymbol{I}\right)=0 \\
\sum_{i=1}^{n}\left|A_{l i} P_{i, i n}^{s}\right| \leq F_{l, \max }, \quad l=1,2, \cdots b \\
\boldsymbol{I} \in(0,1), \quad P_{i, t}^{s} \geq 0
\end{gathered}
$$

In the functions, the superscrip $s$ is the wind power plant limit scenario flag. And when it's the upper limit of wind confidence interval $s=1$. When it's the lower limit $s=2 . \quad \boldsymbol{P}^{s}$ is scheduling thermal output on the limitation scenario(MW). $P_{i, t}^{s}$ is output power for thermal unit $i$ at time $t$ on the limitation scenario(MW). $P_{w j, t}^{s}$ is output power for wind power unit $i$ at time $t$ on the limitation scenario(MW). $P_{i, i n}^{s}$ is the net injection power on node $i$ on the limitation scenario(MW).

Function (4-a) is power balancing constraint and function (4-b) is output constraint on the limitation scenario. Functions (4-c), (4-d) are similar in types, the difference is unit continuous variables in functions (4-c) and (4-d) have superscribe s, which shows wind power limitation scenario.

- Constraints on coupled cooridinate capacity base on confidence interval

In the power system with wind power generation, the most challenge occurs when output ranges through the upper and lower limit within a short time. Other types of generation units should have sufficient coordinate capacity to ensure power balance in the real time running.

Equivalent load confidence interval can be get according to the wind power forecast confidence interval. Former research [21] focuses on the combined unit commitment problem of wind, thermal and pumped storage generation units. It provides a system coordinate capacity base on thermal and pumped storage power limitation scenario. When wind power ranges beyond prediction, the coordinate capacity of thermal and pumped storage units at time t- 1 makes it even that total generation power at time $\mathrm{t}$ can balance the equivalent load. In this way the reliability of unit commitment can be improved.

Thermal output should be able to climb up to the upper equivalent load from the lower value through adjacent time intervals and climb down to lower equivalent load form upper value in regardless of pump strorage generation. By analyzing the coordinate capacity of thermal output throughout the confidence interval of wind forecast, the confidence interval of coupled coordinate capacity constraints are:

$$
\left\{\begin{aligned}
d_{t-1 \_t}^{2} & =\sum_{i=1}^{N}\left[\min \left\{P_{i, t-1}^{2}-P_{i, \min }, D_{i}\right\} I_{i, t-1} I_{i, t}+\right. \\
& \left.P_{i, \min } v_{i, t}-P_{i, \min } u_{i, t}\right] \\
d_{t-1 \_t}^{2} \geq & d L_{t-1}^{2}-d L_{t}^{1}
\end{aligned}\right.
$$




$$
\left\{\begin{aligned}
r_{t-1 \_t}^{1}= & \sum_{i=1}^{N}\left[\min \left\{P_{i, \max }-P_{i, t-1}^{1}, R_{i}\right\} I_{i, t-1} I_{i, t}\right. \\
& \left.+P_{\mathrm{i}, \min } u_{i, t}-P_{i, \min } v_{i, t}\right] \\
r_{t-1 \_t}^{1} \geq & d L_{t}^{2}-d L_{t-1}^{1}
\end{aligned}\right.
$$

and

$$
d L_{t}^{s}=L_{t}-\sum_{j=1}^{W} P_{w j, t}^{s}
$$

In the functions, the superscrip s is the wind power plant limit scenario flag. And when it's the upper limit of wind confidence interval $s=1 . d L_{t-1}^{1}$ is the lower limit of equivalent load, and $d L_{t}^{2}$ is the upper limit(MW). W is the number of wind power plants. $L_{\mathrm{t}}$ is the predicted load at time $\mathrm{t}(\mathrm{MW}) . P_{w j, t}^{s}$ is wind power output in the scenario of $s(\mathrm{MW}) . r_{t-1, t}^{1}$ is system's upper coordinate capacity at time $\mathrm{t}-1$ when the wind power output equals the upper limit of confidence interval and $d_{t-1 \_t}^{2}$ is the upper capacity at the lower confidence interval limitation. $I_{i, t}$ is the running state of thermal units. $P_{i, t-1}^{2}$ and $P_{i, t}^{2}$ are respectively the scheduling output of themal units at time t-1 and at time t. $P_{i, \max }$ is the upper output limit of thermal units and $P_{i, \min }$ is the lower limit. $R_{i}$ and $D_{i}$ are respectively the upper and lower ramping constraints of units.

\section{Example}

An example is used here to prove the efficiency of the unit commitment model above. With the analysis of the example, some conclusions might be researched: 1) Influence of choices of confidence interval on the reliability of unit commitment and the generation cost. 2) Consumption of wind power in a random wind scenario. 3) Completion rate of bilateral transactions and Sangong power and the distribution of contract reduction among generation plants. 4) Influence of power flow constraints on contract execution. 5) Interactive influences among new energy consumption, transmission congestion and contract execution.

\section{Example Discribition.}

In this example, there are three thermal generation plants and one equivalent wind power generation plant. The total capacity of thermal plants is $1200 \mathrm{MW}$ and the capacity of wind plant is $150 \mathrm{MW}$. The percentage of wind capacity is around 11\%. The structure of the system is shown in Fig. 1 and the parameter of thermal units are shown in Table.1. Data of wind power and load forecast is shown in appendix A and data of contracts and reduction factor of plants is shown in Table.2.

\section{Example Result Analysis.}

- Unit Commitment, Generation Cost and Contract Reduction.

Assume the error of wind power prediction is distributed in sub-index. Choose different confidence intervals according to the wind power forecast calculation, unit commitment can be solved respectively. The result is shown in Table 3.

\begin{tabular}{|c|c|c|c|c|c|c|c|c|c|c|c|}
\hline \multirow{2}{*}{ Plants } & \multirow{2}{*}{ Units } & \multirow{2}{*}{$\begin{array}{c}\text { Upper } \\
\text { Output/MW }\end{array}$} & \multirow{2}{*}{$\begin{array}{c}\text { Lower } \\
\text { Output/MW }\end{array}$} & \multirow{2}{*}{$\begin{array}{c}\text { Ramping } \\
\text { Capacity/(MW/h) }\end{array}$} & \multirow{2}{*}{$\begin{array}{l}\text { Lower } \\
\text { Startup } \\
\text { Time/h }\end{array}$} & \multirow{2}{*}{$\begin{array}{c}\text { Lower } \\
\text { Stop } \\
\text { Time/h }\end{array}$} & \multicolumn{3}{|c|}{ Coal Consumption Rate } & \multirow{2}{*}{$\begin{array}{c}\text { Startup } \\
\text { Cost/ } \\
¥\end{array}$} & \multirow{2}{*}{$\begin{array}{c}\text { Coal } \\
\text { Price/( } ¥ / G J)\end{array}$} \\
\hline & & & & & & & $\mathrm{a} / \mathrm{GJ}$ & $\mathrm{b} /(\mathrm{GJ} / \mathrm{MWh})$ & $\mathrm{c} /\left(\mathrm{GJ} / \mathrm{MW}^{2} \mathrm{~h}\right)$ & & \\
\hline \multirow{3}{*}{ A } & 1 & 100 & 10 & 50 & 4 & 4 & 176.9 & 13.5 & 0.0004 & 100 & 7.7467 \\
\hline & 2 & 200 & 50 & 60 & 2 & 3 & 129.9 & 32.6 & 0.0004 & 300 & 7.7418 \\
\hline & 3 & 300 & 60 & 30 & 1 & 1 & 137.4 & 17.6 & 0.0004 & 100 & 7.7424 \\
\hline $\mathrm{B}$ & 4 & 100 & 20 & 30 & 2 & 2 & 125.8 & 20 & 0.0003 & 200 & 7.7436 \\
\hline \multirow{3}{*}{$\mathrm{D}$} & 5 & 120 & 20 & 40 & 1 & 1 & 140.6 & 30 & 0.0006 & 200 & 7.7421 \\
\hline & 6 & 180 & 30 & 50 & 2 & 1 & 145.7 & 40 & 0.0006 & 300 & 7.7436 \\
\hline & 7 & 200 & 40 & 40 & 1 & 2 & 156.9 & 10 & 0.0006 & 100 & 7.7410 \\
\hline
\end{tabular}

Tab.1 Parameters of thermal units 


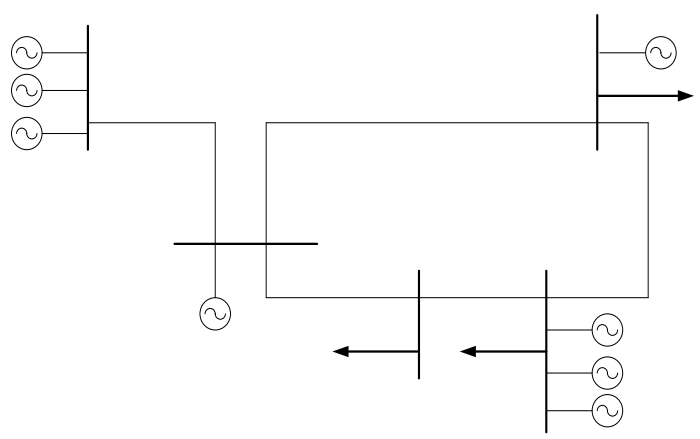

Fig. 1 Five-node test syste

Tab.2 Contract information of power plants and contract reduction factor

\begin{tabular}{ccccc}
\hline \multirow{2}{*}{ Plants } & Bilateral Contracts/MW*h & Sangong Contracts/MW*h & Total & Reduction Factor \\
\hline A & 6447 & 1611.75 & 8058.75 & 0.5 \\
B & 1074.5 & 268.63 & 1343.13 & 0.083 \\
D & 5372.5 & 1343.13 & 6715.63 & 0.417 \\
\hline
\end{tabular}

The reliability of unit commitment is examined by 200 random wind power scenarios. If the unit commitment solved by a given confidence interval can consume all wind power and ensure the security of power grid on a random scenario, then there is a feasible solution in this condition. And this unit commitment is reliable. Otherwise, the unit commitment is unreliable. The examine result of unit commitment and generation cost within different confidence intervals is shown in Table 4. And the result of contract reduction distribution among plants is shown in Table 5.

Tab.3 Unit commitments

\begin{tabular}{ccccc}
\hline \multirow{2}{*}{ Units } & \multicolumn{4}{c}{ Unit Commitment of Different Confidence Interval } \\
\cline { 2 - 5 } & $50 \%$ & $80 \%$ & $90 \%$ & $98 \%$ \\
\hline G1 & all day & all day & all day & all day \\
G2 & $1-4$ & $1-4$ & all day & $1-4$ \\
G3 & all day & all day & all day & all day \\
G4 & $1-19$ & all day & all day & all day \\
G5 & all day & all day & all day \\
G6 & $1-2,15-22$ & $1-2,13-21$ & $1-2,12-22$ & $1-3,10-22$ \\
G7 & all day & all day & all day \\
\hline
\end{tabular}

Tab.4 Generation cost and the reliability of unit commitments

\begin{tabular}{ccc}
\hline Confidence Interval & Generation Cost/ $¥$ & Reliability of Unit Commitment/\% \\
\hline $50 \%$ & 2086978.8 & 62.5 \\
$80 \%$ & 2093209.1 & 79 \\
$90 \%$ & 2100114.4 & 93 \\
$98 \%$ & 2114857.3 & 99.5 \\
\hline
\end{tabular}

-Example Result Analysis

(1) Influence of confidence interval on reliability of unit commitment, generation economy and transaction execution.

The fluctuation interval of wind power relates to the given wind power confidence interval. And the larger of interval range, the more of possible output situation for wind power, and the more of coordinate capacity for the unit commitment. By combining the results of Table 3 and Table 4, the reliability of unit commitment increases as the range of forecast interval increases. There is also an increase in the boot time of units and the generation cost. If the chosen confidence interval is $98 \%$, the reliability of unit commitment can reach 99\%.

(2)validity testification of wind power accommodation under various confidence interval.

In order to testify the validity of the model, it's need to verify the reliability of unit commitment to the output curve under wind power scenarioes which are all located in the confidence interval. Preliminary study in the thesis [21] has testified the validity of confidence interval in the joint optimization of wind power, thermal power and pumped storage power. In this paper, in order to testify the impact of day-ahead balance model of electric power and electric volume to wind power accommodation, in which model the bilateral transactions are included, confidence interval is set as $98 \%$ to testify its validity in which case there is no feasible solution in that wind power scenario. 
Figure 2 is random choose of the unsolved scenario and the verified scenario. The verified scenario is to modify the data in over-limit duration according to the above and below limit of the confidence interval insuring the verified wind power curve is totally located in the inner area of the confidence interval. Based on the verified scenario, the unit commitment is confirmed to asure the real-time power balance accommodating all the wind power and the system operation cost is 2158979.5 yuan.

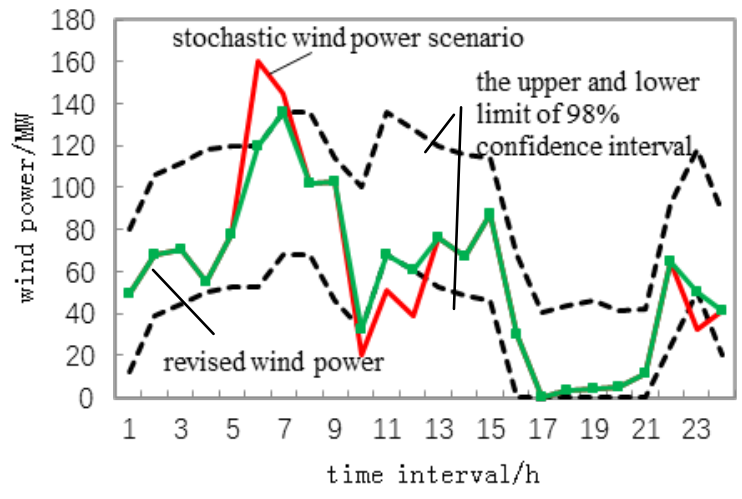

Fig. 2 A validation scenario without solution and the modified scenario

(3)impact of power flow to transaction execution

Considering the volatility and contract electric volume execution, the confidence interval is chosen as $98 \%$ and the power transfer factor of line A-E to nodes B, C, D and E are -0.112 、 $-0.2629 、-0.321$ and -0.4805 , the impact of power flow constraints' existance on the contract electric volume execution is compared, as Tab 5.

Tab.5 The impact of power flow to contract execution

\begin{tabular}{ccc}
\hline Case & Contract reductions/MW & cost/yuan \\
\hline \multirow{3}{*}{ no power flow restrictions } & \multirow{2}{*}{1471.2300} & A: 735.615 \\
& B: 122.1121 & 1938727.5 \\
D: 613.5029 & 2114857.3 \\
\hline \multirow{2}{*}{ A-E power flow restriction } & A: 1521.3547 \\
& B: 252.54487 \\
D: 1268.8098 & \\
\hline
\end{tabular}

Through comparison in table 5, when the line congestion exists, contract reduction adds by 1.07 times than it of the case without power flow constraints, generation cost adds by $8.9 \%$, power flow restrictions are not good for contract execution.

(4) mutual influence of wind power location, power flow constraints and contracts execution

As the location of wind power has various impacts on the power flow, it also influences the contract volume execution.

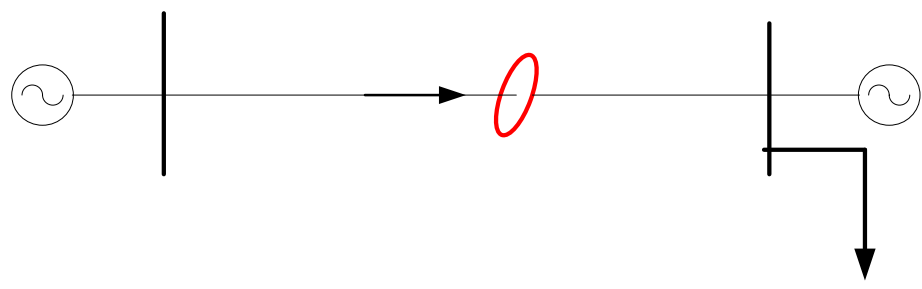

Fig. 3 The location of wind power of two cases

As in figure 3, wind farm is located at node E in case 1 and at node $\mathrm{A}$ in case 2, considering wind power accommodation and power flow constraints, the electric volume execution and its generation cost in each case are compared whose results are in Tab 6. 
Tab.6 The impact of locations of wind power to contract execution

\begin{tabular}{ccc}
\hline Case & Contract reductions/MWh & total contract reductions/MWh \\
\hline \multirow{3}{*}{ no wind power } & A: 2171.371 & \multirow{2}{*}{4342.74264} \\
& B: 360.448 & \\
& D: 1810.924 & 3042.70937 \\
\hline \multirow{2}{*}{ Case1-E } & A: 1521.355 & \\
& B: 252.545 & \multirow{2}{*}{ D: 1268.890} \\
Case2-A 点 & A: 3499.072 & \\
& B: 580.846 & \\
\hline \multirow{2}{*}{ D: 2918.226} & \\
\hline
\end{tabular}

As in Table 6, when the wind farm is located at node E, contracts execution reduction gets its minimum, while when the wind farm is located at node A, contracts execution reduction gets its maximum. This is because when the scale of wind power is relatively small, wind power arouses reverse power flow at node $\mathrm{E}$ which helps improving the contract volume execution, and the contract conduction volume is $42.73 \%$ lower than the no-wind-power case. By contrast, when wind power is located in node $\mathrm{A}$, it adds to the forward power flow which occupies the thermal power transfer passage, influencing the contract electric volume execution, and the contract conduction volume is $61.15 \%$ higher than the no-wind-power case.

\section{Conclusions}

When the bilateral contracts are electric volume ones, as the bilateral contracts settlement is only related with electric volume, they have no essential difference with the justice dispatch electric volume. Thus, the bilateral and justice contracts are combined to deal with for unified modeling which simplifies the complexity of the problem. What's more, the execution priority of the two could be determined by rational settlement rules.

The multi-objective optimization of day-ahead unit commitment problem is so complicated considering the power system security, wind power accommodation priority, execution of bilateral contracts and justice dispatch contracts and the economy of power generation. This paper adopts the joint optimization objective of contracts execution and generation economy in which the contracts execution has a higher rank than the generation economy. The confidence interval approach is adopted to deal with the uncertainty of wind power based on the confidence interval's up and down limits' coupling constraints. The execution of contract volume is guaranteed by setting the objective and constraints.

In this case example, to guarantee the reliability of unit commitment above 99\%, the confidence interval should be chosen as $98 \%$. In details, the confidence interval must be determined by various kinds of power source capacity ratio and fluctuation characteristics of wind power. By the effectiveness testified model of confidence interval, it can be guaranteed that wind power can be totally accommodated when the wind power output in practical operation is any of curves inside the confidence interval. The difference of the confidence intervals would influence the accommodation of wind power. Contracts execution under various confidence interval is only equal to that considering forcast wind power only. When the operation security constraints are broken, contract volume would be reduced, in which case the plants reduce their contract volume by each plant's contract reduction factor insuring the faireness between the plants. Contract execution is influenced by the power flow constraints. In detail, when wind power is considered and power flow constraints exists, the reduction volume of contracts amounts by 1.07 by contrast with the one with no power flow constraints and generation cost adds by $8.9 \%$. When the scale of wind power is small and power flow constraints exist, the different location of wind power has different influence on the contract execution. If wind power adds to the forward power flow of the congestion lines which is bad for contracts execution,the reduction volume would add by $61.15 \%$ compared with the no-wind-power case. While, if wind power arouses reverse power flow of the congestion lines which is good for contracts execution,the reduction volume would drop by $42.73 \%$ compared with the no-wind-power case.

When bilateral contracts are electric power ones, their settlement is related with the electric 
power curve stipulated in the contracts, the method modeling and processing would be different. As restriction of the paper, the optimization modeling on bilateral electric power contracts may be studied in the following papers.

\section{Acknowledgments}

This work was financially supported by State Grid Corporation Science and Technology Project (DZ71-15-086) fund.

\section{References}

[1] Kashif Imran, Ivana Kockar. A technical comparison of wholesale electricity markets in North America and Europe[J]. Electric Power Systems Research,108(2014),59-67.

[2] State electricity regulatory commission. The electric power market of America. China electric power press.2005.

[3] Daniel S.Kirschen,Goran Strbac. Fundamentals of Power System Economics.Wiley,2004.

[4] State electricity regulatory commission.Europe, Australia electric power market. China electric power press, 2005.

[5] Peng Tao, Xia Qing, Jiang Jianjian, Kang Chongqing. A nalysis of economic mechanism of two oregional electricity market model[J]. Automation of Electric Power Systems, 2004,28(07):20-23+29(in Chinese).

[6] Yao Jiangang,Tang Jie,Li Xiquan,Zhang Binghui,Mu Lei. Research on bidding mode in a generation-side power market[J]. Proceedings of the CSEE, 2004(05) (in Chinese).

[7] Ding Junwei, Xia Qing, Hu Yang, Chen Zhi, Kang Chongqing, Shen Yu.A new model for gradually establishing competitive generation market[J]. Proceedings of the CSEE, 2003,03:10-15(in Chinese).

[8] Li Canbing, Kang Chongqing, Xia Qing, Huang Yonghao, Meng Yuanjing, Shang Jincheng, He Nanqiang. Study on trading mechanism of regional electricity market[J]. Power system technology. 2004,07:34-39(in Chinese). .

[9] Hamed Kebriaei,Vahid Johari Majd .A simultaneous multi-attribute soft-bargaining design for bilateral contracts[J]. Expert Systems with Applications, 36 (2009) ,4417-4422.

[10]Zhang Xian, Wang Xifan, Chen Haoyong, Wang Jianxue. Survey of bilateral contracts in power market[J]. Electric power automation equipment., 2003,11:77-86(in Chinese).

[11]Zhang Xian, Wang Xifan, Wang Jianxue, Hu Zechun. Application of block trading in bilateral market [J].Automation of electric power systems, 2004, 11:13-16(in Chinese).

[12]Zou Xiaoyan. The bargain game and power energy allocation of bilateral contract in power market[J]. Demand side management, 2007, 04:34-36(in Chinese). .

[13]Wen Tian, Du Songhuai, Su Juan. Available transfer capability determination considering bilateral exchange[J]. Transactions of the Chinese Society of Agricultural Engineering, 2013, 29(Supp.1): 161-166(in Chinese).

[14]Han Jinrui, Zeng Ming, Duan Jinhui, Li Na. The bilateral electricity market modes of typical countries[J]. East china electric power, 2013,01:17-23(in Chinese).

[15]Zeng Ming, Liu Chao, Duan Jinhui, Li Na. The bilateral electricity market modes of typical countries and our reference from america and nordic[J]. East china electric power, 2013, 
41(1):5-10(in Chinese).

[16]Zeng Ming, Duan Jinhui, Li Na, Dong Jun. The experience of brithish bilateral electricity market modes[J]. East china electric power, 2013, 41(1):1-4(in Chinese).

[17]Zeng Ming, Cheng Jun, Duan Jinhui, Dong Jun, Liu Chao. The bilateral electricity market modes of typical countries and our reference from australia and russia [J]. East china electric power, 2013, 01:11-16(in Chinese).

[18]Liu Fang,Pan Yi,Liu Hui,Ding Qiang,Wang Zhiming.

Research on Piecewise Exponential distribution model of wind power forecasting error[J]. Automation of Electric Power Systems,2013,18:14-19(in Chinese).

[19]Liu Fang, Pan Yi, Yang Junfeng, Zhou Jieying, Zhou Jingyang, etc. Unit commitment model for combined optimization of wind power-thermal power-pumped storage hydro[J]. Proceedings of the CSEE, 2015, 04:766-775(in Chinese).

\section{APPENDIX A}

Tab.A Forecast load and forecast wind power

\begin{tabular}{|c|c|c|c|c|c|c|c|c|c|c|c|}
\hline hour & $\begin{array}{l}\text { load ( Lc= } \\
\text { Ld } \\
=\mathrm{Le}) / \mathrm{MW}\end{array}$ & $\begin{array}{c}\text { forcast wind } \\
\text { power } \\
\text { /MW }\end{array}$ & hour & $\begin{array}{l}\text { load }(\mathrm{Lc}= \\
\text { Ld } \\
=\mathrm{Le}) / \mathrm{MW}\end{array}$ & $\begin{array}{c}\text { forcast wind } \\
\text { power } \\
\text { /MW }\end{array}$ & hour & $\begin{array}{l}\text { load ( Lc= } \\
\text { Ld } \\
=\mathrm{Le}) / \mathrm{MW}\end{array}$ & $\begin{array}{c}\text { forcast wind } \\
\text { power } \\
\text { /MW }\end{array}$ & hour & $\begin{array}{l}\text { load ( Lc= } \\
\text { Ld } \\
=\mathrm{Le}) / \mathrm{MW}\end{array}$ & $\begin{array}{c}\text { forcast wind } \\
\text { power } \\
\text { /MW }\end{array}$ \\
\hline 1 & 206.40 & 44 & 7 & 224.46 & 100 & 13 & 242.06 & 84 & 19 & 218.66 & 10 \\
\hline 2 & 211.78 & 70.2 & 8 & 230.13 & 100 & 14 & 241.20 & 80 & 20 & 212.45 & 5 \\
\hline 3 & 211.56 & 76 & 9 & 227.85 & 78 & 15 & 242.29 & 78 & 21 & 214.44 & 6 \\
\hline 4 & 212.24 & 82 & 10 & 227.07 & 64 & 16 & 229.26 & 32 & 22 & 227.71 & 56 \\
\hline 5 & 213.02 & 84 & 11 & 242.87 & 100 & 17 & 220.00 & 4 & 23 & 227.68 & 82 \\
\hline 6 & 214.83 & 84 & 12 & 242.70 & 92 & 18 & 215.58 & 8 & 24 & 216.25 & 52 \\
\hline
\end{tabular}

